\title{
Dynamic transition and Shapiro step melting in a frustrated Josephson-junction array
}

\author{
Jong Soo Lim, ${ }^{1}$ M.Y. Choi,,${ }^{1,2}$ J. Choi, ${ }^{3}$ and Beom Jun Kim ${ }^{4}$ \\ ${ }^{1}$ Department of Physics, Seoul National University, Seoul 151-747, Korea \\ ${ }^{2}$ Korea Institute for Advanced Study, Seoul 130-722, Korea \\ ${ }^{3}$ Department of Physics, Keimyung University, Daegu 704-701, Korea \\ ${ }^{4}$ Department of Molecular Science and Technology, Ajou University, Suwon 442-749, Korea
}

\begin{abstract}
We consider a two-dimensional fully frustrated Josephson-junction array driven by combined direct and alternating currents. Interplay between the mode locking phenomenon, manifested by giant Shapiro steps in the current-voltage characteristics, and the dynamic phase transition is investigated at finite temperatures. Melting of Shapiro steps due to thermal fluctuations is shown to be accompanied by the dynamic phase transition, the universality class of which is also discussed.

PACS numbers: 74.81.Fa, 74.25.Nf, 05.40.-a
\end{abstract}

Driven systems with many degrees of freedom are ubiquitous in nature and exhibit a rich variety of dynamic behavior as the driving or the temperature is varied. Here the usual equilibrium concepts are not applicable, making the study of nonequilibrium transitions in such driven systems very important and highly nontrivial. A prototype system is provided by the Josephson-

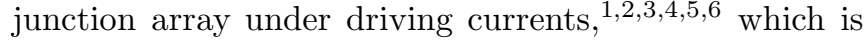
also closely related to, e.g., moving vortex lattices and sliding charge density waves $\stackrel{7.8}{ }$ In equilibrium the fully frustrated Josephson-junction array (FFJJA), as an experimental realization of the fully frustrated $X Y$ model with the $\mathrm{U}(1) \times \mathrm{Z}_{2}$ symmetry, has been a source of controversy as to whether a $Z_{2}$ transition and a $U(1)$ transition occur at the same temperature 9.10.11.12 $^{9}$ In the presence of direct driving currents, the transition temperatures in general reduce, ${ }_{,}^{2}$ approaching zero as the current is increased toward the critical current; beyond it, the $\mathrm{Z}_{2}$ transition occurs at a substantially lower temperature than the $\mathrm{U}(1)$ transition ${ }^{4}$ On the other hand, when the driving current has only the ac component, the FFJJA has been shown to exhibit a dynamic $Z_{2}$ transition, which belongs to the same universality class as the equilibrium $\mathrm{Z}_{2}$ transition $\underline{\underline{6}}$

The remaining case is the FFJJA driven by combined direct and alternating currents, which is well known to display quantized voltage plateaus, called giant Shapiro steps, in the current-voltage characteristics at zero temperature 13 This is a manifestation of mode locking in response to the driving current, associated with topological invariance of the system. 14 However, the issue of the FFJJA driven by combined direct and alternating currents has not been addressed from the viewpoint of the dynamic phase transition, which requires analysis of the system at finite temperatures.

This work investigates how such a mode locking phenomenon changes into the dynamic transition, as the temperature is raised from zero. To describe the dynamic transition associated with topological invariance, we introduce the dynamic order parameter and investigate its behavior with the driving amplitude. The zerotemperature behavior of the dynamic order parameter is discussed in the framework of the topological invariance of Shapiro steps. As the temperature is raised from zero, the Shapiro steps are shown to melt, which is accompanied by the dynamic phase transition. From the detailed behavior of the dynamic order parameter at finite temperatures, we construct the dynamic phase diagram on the plane of the temperature and the direct current, and examine nature of the transition.

To begin with, we consider the equations of motion for phase angles $\left\{\phi_{i}\right\}$ of the superconducting order parameters in grains, which form an $L \times L$ square lattice. Within the resistively-shunted-junction model under the fluctuating twist boundary conditions,,$\frac{15}{2}$ they read:

$$
\sum_{j}^{\prime}\left[\frac{d \widetilde{\phi}_{i j}}{d t}+\sin \left(\widetilde{\phi}_{i j}-\mathbf{r}_{i j} \cdot \boldsymbol{\Delta}\right)+\eta_{i j}\right]=0,
$$

where the primed summation runs over the nearest neighbors of grain $i$ and the thermal noise current $\eta_{i j}$ satisfies $\left\langle\eta_{i j}(t) \eta_{k l}\left(t^{\prime}\right)\right\rangle=2 T \delta\left(t-t^{\prime}\right)\left(\delta_{i k} \delta_{j l}-\delta_{i l} \delta_{j k}\right)$ at temperature $T$. We have used the abbreviations $\widetilde{\phi}_{i j} \equiv \phi_{i}-\phi_{j}-A_{i j}$ and $\mathbf{r}_{i j} \equiv \mathbf{r}_{i}-\mathbf{r}_{j}$ with $\mathbf{r}_{i}=\left(x_{i}, y_{i}\right)$ denoting the position of grain $i$. Note that $\mathbf{r}_{i j}$ for nearest neighboring grains becomes a unit vector with the lattice constant set equal to unity. We have also written the energy and the time in units of $\hbar i_{c} / 2 e$ and $\hbar / 2 e R i_{c}$, respectively, with the critical current $i_{c}$ and the shunt resistance $R$ of a single junction. The dynamics of the twist variable $\boldsymbol{\Delta} \equiv\left(\Delta_{x}, \Delta_{y}\right)$ is governed by the equations

$$
\begin{aligned}
& \frac{d \Delta_{x}}{d t}=\frac{1}{L^{2}} \sum_{\langle i j\rangle_{x}} \sin \left(\widetilde{\phi}_{i j}-\Delta_{x}\right)+\eta_{\Delta_{x}}-I_{d c}-I_{a c} \sin \Omega t \\
& \frac{d \Delta_{y}}{d t}=\frac{1}{L^{2}} \sum_{\langle i j\rangle_{y}} \sin \left(\widetilde{\phi}_{i j}-\Delta_{y}\right)+\eta_{\Delta_{y}}
\end{aligned}
$$

where $\sum_{\langle i j\rangle_{\mu}}$ denotes the summation over all nearest neighboring pairs in the $\mu(=x, y)$ direction, $\eta_{\Delta_{\mu}}$ satisfies $\left\langle\eta_{\Delta_{\mu}}(t) \eta_{\Delta_{\mu^{\prime}}}\left(t^{\prime}\right)\right\rangle=\left(2 T / L^{2}\right) \delta\left(t-t^{\prime}\right) \delta_{\mu \mu^{\prime}}$, and the combined direct and alternating currents $I_{d c}+I_{a c} \sin \Omega t$ are injected in the $x$ direction. In the Landau gauge, the bond angle $A_{i j}$, given by the line integral of the vector potential, vanishes for $\mathbf{r}_{j}=\mathbf{r}_{i}+\hat{\mathbf{x}}$ and takes the value $\pi x_{i}$ for $\mathbf{r}_{j}=\mathbf{r}_{i}+\hat{\mathbf{y}}$. 
At zero temperature the FFJJA driven by combined direct and alternating currents displays integer, fractional, and subharmonic giant Shapiro steps ${ }^{13.14}$ The system evolves periodically in time with the topological invariant period, characteristic of each voltage step. Time evolution of the phase configuration shows that the system visits periodically various accessible states consisting of the ground states, the transient states, and other dynamically accessible states of the purely dynamic origin $\underline{16}$ To characterize such dynamic behavior associated with the $\mathrm{Z}_{2}$ symmetry in the FFJJA, we consider the chirality

$$
C(\mathbf{R}, t) \equiv \operatorname{sgn}\left\{\sum_{\mathbf{P}} \sin \left[\widetilde{\phi}_{i j}(t)-\mathbf{r}_{i j} \cdot \boldsymbol{\Delta}(t)\right]\right\}
$$

and the staggered magnetization

$$
m(t) \equiv \frac{1}{L^{2}} \sum_{\mathbf{R}}(-1)^{x_{i}+y_{i}} C(\mathbf{R}, t)
$$

where $\sum_{\mathbf{P}}$ denotes the directional plaquette summation of links around the dual lattice site $\mathbf{R} \equiv \mathbf{r}_{i}+(1 / 2)(\hat{\mathbf{x}}+\hat{\mathbf{y}})$.

Figure 1 1 exhibits the zero-temperature time evolution of the staggered magnetization on various voltage steps, where $\bar{V}$ denotes the time-averaged voltage in units of

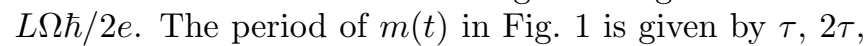
and $4 \tau$ on steps $\bar{V}=1,1 / 2$, and $1 / 4$, respectively. Here we have considered the FFJJA of size up to $L=24$, and integrated numerically the equations of motion in Eqs. (11) and (2) via the modified Euler method, using time steps of size $\Delta t=0.01$. The values of $\Delta t$ have been varied, only to give insignificant difference. Typically, data have been averaged over 1000 driving periods of the (alternating) current, after the initial 500 periods discarded; this has turned out sufficient for reaching appropriate stationarity.

At high temperatures thermal fluctuations are so strong that the influence of the driving current and the lattice potential can be neglected, leading to random fluctuations of $m(t)$. We thus expect a phase transition between the dynamically ordered phase and the disordered one as the temperature is varied. To describe such a dynamic phase transition, we introduce the dynamic order parameter, defined to be the staggered magnetization averaged over $n$ periods of the (alternating) current:

$$
Q \equiv \frac{\Omega}{2 \pi n}\left|\int_{t_{0}}^{t_{0}+n \tau} m(t) d t\right|
$$

with $\tau \equiv 2 \pi / \Omega$. On each Shapiro step, we consider the topologically invariant period ${ }^{16}$ to choose the proper integer $n$. Specifically, on the $1 / 2 q$ step we choose $n=q$, avoiding the null order parameter. In general, the dynamic order parameter in Eq. (5) depends on $t_{0}$ and henceforth the maximum value is defined to be the dynamic order parameter: $Q \equiv \max _{t_{0}} Q\left(t_{0}\right)$.

Figure 2 shows the ensemble average $\langle Q\rangle$ of the dynamic order parameter as a function of the temperature

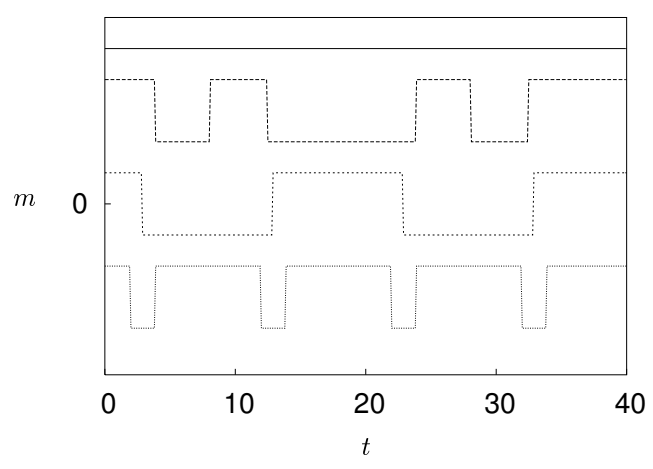

FIG. 1: Time evolution of the staggered magnetization $m(t)$ at zero temperature. The amplitude and the frequency of the ac component are $I_{a c}=1.0$ and $\Omega / 2 \pi=0.1$, respectively. The dc component is given by $I_{d c}=0.05,0.177,0.40$, and 0.75 from the top to the bottom, corresponding to the average voltage $\bar{V}=0,1 / 4,1 / 2$ and 1 . The period of $m(t)$ is $\tau(\equiv$ $2 \pi / \Omega=10), 2 \tau$, and $4 \tau$ on the steps $\bar{V}=1,1 / 2$ and $1 / 4$, respectively. For clarity, data for $\bar{V}=0$ and $1 / 4$ have been shifted upward, while those for $\bar{V}=1$ shifted downward.

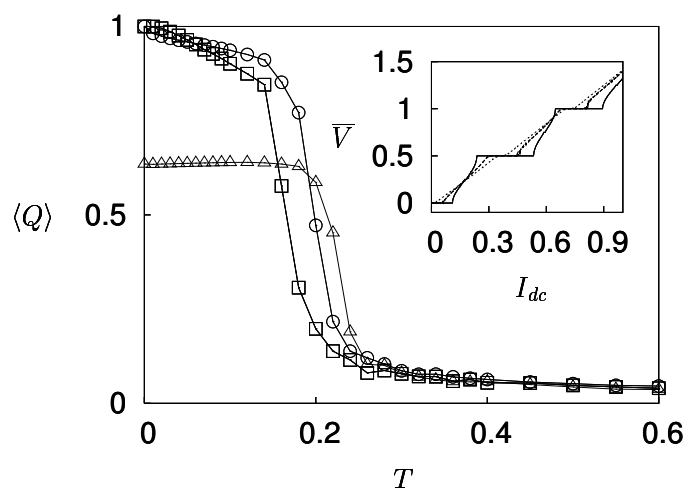

FIG. 2: Dynamic order parameter as a function of the temperature in the system of size $L=24$ for $I_{d c}=0.04(\square), 0.4(\bigcirc)$, and $0.75(\triangle)$, corresponding to $\bar{V}=0,1 / 2$, and 1 , respectively. Lines are merely guides to eyes. Inset: Time averaged voltage $\bar{V}$ versus the dc component $I_{d c}$ at temperature $T=0,0.1$, and 0.24 . As $T$ is raised, the voltage plateaus tend to melt.

$T$ at various values of the dc component $I_{d c}$. Manifested is the presence of a dynamic phase transition, separating the dynamically ordered phase at low temperatures and the disordered phase at high temperatures. In all the three cases in Fig. 2] corresponding to the voltage steps $\bar{V}=0,1 / 2$, and 1 , the dynamic order parameter increases gradually as the temperature is lowered from high temperatures. Subsequently, it grows rapidly, eventually saturating to the zero-temperature value. Further, it is also observed that cooling and heating curves for the dynamic order parameter exhibit appreciable hysteresis at low temperatures (not shown here), which arises from the asymmetry of the lattice potential induced by the dc component of the driving current and the resulting 


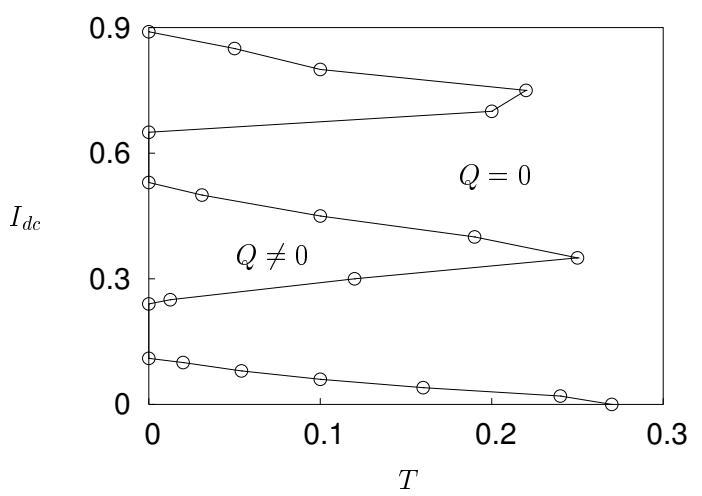

FIG. 3: Dynamic phase diagram on the $T-I_{d c}$ plane. The phase boundaries are determined by the crossing points of Binder's cumulants for size $L=8,16$, and 24 . The areas enclosed by the boundaries specify dynamically ordered phases characterized by $Q \neq 0$. Lines are merely guides to eyes.

anisotropy, similarly to the dc driven case $\stackrel{4}{\underline{4}}$ Such behavior does not appear in the system driven by alternating currents only (without the dc component).

The Shapiro step originates from the interplay between the period of the external driving current and the period emerging from the intrinsic dynamics of the system. Such mode locking behavior present in the low temperature regime is expected to smear out as the thermal fluctuations become strong. Indeed the Shapiro steps are observed to melt as the temperature is raised, disappearing eventually at sufficiently high temperatures (see the inset of Fig. (2). To probe the relation between this melting behavior and the dynamic phase transition, we first estimate the transition temperature accurately via Binder's cumulant $\underline{17}$

$$
U_{L}=1-\frac{\left\langle Q^{4}\right\rangle}{3\left\langle Q^{2}\right\rangle^{2}}
$$

which takes the value $2 / 3$ at $T=0$, vanishes in the hightemperature limit, and becomes size-independent at the transition temperature. The unique crossing point of $U_{L}$, independent of the system size $L$, for given $I_{d c}$ thus yields the corresponding data point for the phase boundary on the $T-I_{d c}$ plane in Fig. 3 The areas enclosed by the resulting phase boundaries specify the dynamically ordered phase, characterized by $Q \neq 0$, for $\bar{V}=0,1 / 2$, and 1 from the bottom to the top. The transition temperature initially decreases monotonically to zero as the driving amplitude $I_{d c}$ is increased in the region of $\bar{V}=0$. Further increase of $I_{d c}$ drives the system into another ordered region, characterized by $\bar{V}=1 / 2$, where the transition temperature first grows with the driving current, then reduces to zero, and so on.

For comparison, phase boundaries are also estimated independently from melting of the Shapiro steps. Here we define the width of a step as follows: For a given point on a voltage step, we measure the slope of the straight line connecting the point to the middle point of the step and
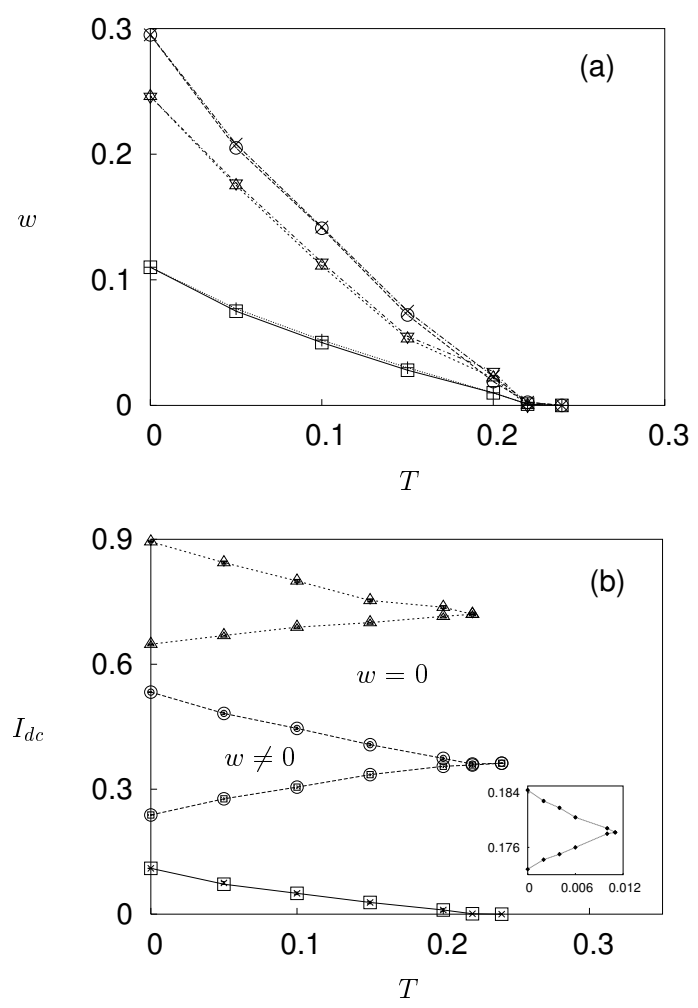

FIG. 4: (a) Width $w$ of the voltage plateau versus the temperature $T$ on the step $\bar{V}=0(\square,+), 1 / 2(\bigcirc, \times)$, and $1(\triangle, \nabla)$, where the two symbols for each step represent the data from two different criteria of the slope: 0.005 and 0.01 , respectively. (b) Dynamic phase diagram on the $T-I_{d c}$ plane, determined by the width of voltage plateaus for size $L=16$. The areas enclosed by the phase boundaries specify dynamically ordered phases with $w \neq 0$. Lines are merely guides to eyes. Inset: Detailed diagram displaying the ordered phase corresponding to the subharmonic voltage step $\bar{V}=1 / 4$, again determined by its width.

consider the point to be located on the step if the slope is less than 0.005. Namely, the step width is determined by the boundary point, for which the slope is given by 0.005 [the use of other values hardly changes the resulting phase boundaries, as shown in Fig. 4(a)]. The step width obtained in this manner diminishes with the temperature and eventually vanishes to zero [see Fig. [4(a)], from which each phase boundary in Fig. 世 (b) has been estimated. The good agreement between Figs. B 3 and 4 (b) manifests the correspondence between the nonzero value of the dynamic order parameter and the finite width of the Shapiro step, thus indicating that the dynamic phase transition accompanies melting of Shapiro steps. It is also expected that there exist additional dynamically ordered phases, associated with the series of subharmonic Shapiro steps ${ }^{16}$ they presumably form Arnold tongue structure $\frac{18}{2}$ in the complete phase diagram. We have thus examined the subharmonic step $\bar{V}=1 / 4$, and indeed found the corresponding ordered phase, shown in the in- 


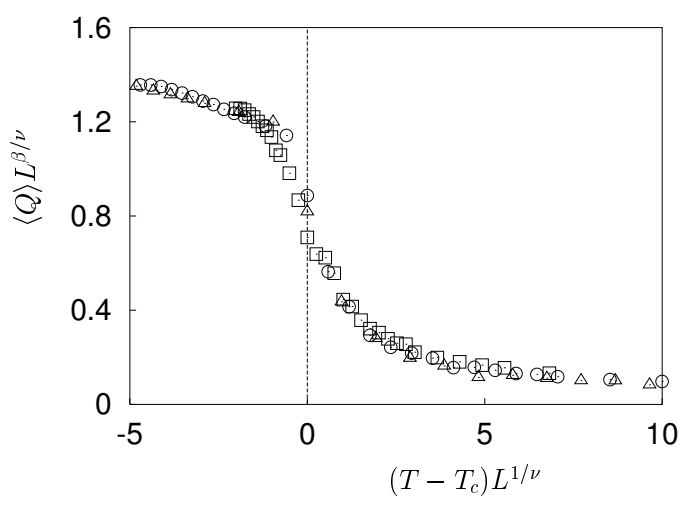

FIG. 5: Scaling plot of the dynamic order parameter for size $L=8(\square), 16(\bigcirc)$, and $24(\triangle)$ at dc component $I_{d c}=0.04$. The fitting has been performed with the critical exponents $\nu=0.82$ and $\beta / \nu=0.11$.

set of Fig. 4(b). Unfortunately, other phases, corresponding to higher-order subharmonic steps, occupy even tinier regions, making it very difficult to locate the boundaries.

We finally study the nature of the transition by means of finite-size scaling for the dynamic order parameter:

$$
\langle Q\rangle=L^{-\beta / \nu} f\left(\left(T-T_{c}\right) L^{1 / \nu}\right) .
$$

In Fig. 5 we plot $\langle Q\rangle L^{\beta / \nu}$ versus $\left(T-T_{c}\right) L^{1 / \nu}$ at $I_{d c}=$ 0.04 (corresponding to $\bar{V}=0$ ), which yields reasonable scaling collapse with the critical exponents $\nu=0.82$ and $\beta / \nu=0.11$. Since these values of the critical exponents agree well with those for the equilibrium $\mathrm{Z}_{2}$ transition in the FF $X Y$ model, ${ }^{3.11}$ we conclude that the dynamic phase transition in the system here belongs to the same universality class as the equilibrium $\mathrm{Z}_{2}$ transition. Similar conclusion was also reached in the FFJJA under weak staggered oscillating fields or uniform alternating currents 5.6 This suggests that the universality class of the transition does not change if $\bar{V}$ remains to be zero (i.e., in the same Arnold tongue). On the other hand, at larger values of $I_{d c}$ corresponding to $\bar{V} \neq 0$, the scaling plot does not collapse well and there still exists ambiguity on the nature of the transition; this appears similar to the dc driven case, where the transition nature changes as the driving current exceeds the critical value. ${ }^{2,4}$

In summary, we have examined the 2D FFJJA driven by combined direct and alternating currents, as a prototype example of driven systems exhibiting rich dynamic behaviors. The relation between melting of the voltage plateaus (Shapiro steps) and the dynamic phase transition has been explored. It has been observed that melting of the Shapiro steps is accompanied by the dynamic phase transition, revealing the correspondence between the two phenomena. We have also examined the nature of the transition, to find, on the zero voltage step, the same universality class as the equilibrium $\mathrm{Z}_{2}$ transition.

This work was supported in part by KOSEF grants R01-2002-000-00285-0 and R14-2002-062-01000-0 as well as by the BK21 Program.
${ }^{1}$ K.K. Mon and S. Teitel, Phys. Rev. Lett. 62, 673 (1989); J.S. Chung, K.H. Lee, and D. Stroud, Phys. Rev. B 40, 6570 (1989); M.Y. Choi and S. Kim, ibid. 44, 10411 (1991).

2 S. Kim and M.Y. Choi, Phys. Rev. B 48, 322 (1993).

3 H.J. Luo, L. Schülke, and B. Zheng, Phys. Rev. Lett. 81, 180 (1998).

4 V.I. Marconi and D. Domínguez, Phys. Rev. Lett. 87, 017004 (2001).

${ }^{5}$ G.S. Jeon, H.J. Kim, M.Y. Choi, B.J. Kim, and P. Minnhagen, Phys. Rev. B 65184510 (2002).

${ }^{6}$ G.S. Jeon, J.S. Lim, H.J. Kim, and M.Y. Choi, Phys. Rev. B 66024511 (2002).

7 C. Reichhardt, C.J. Olson, and F. Nori, Phys. Rev. B 58, 6534 (1998); G. Carneiro, ibid. 66, 054523 (2002).

8 G. Grüner, Density Waves in Solids (Addison-Wesley, Reading, 1994).

9 S. Teitel and C. Jayaprakash, Phys. Rev. B 27, 598 (1983); M.Y. Choi and S. Doniach, ibid. 31, 4516 (1985).

10 J. Lee, J.M. Kosterlitz, and E. Granato, Phys. Rev. B 43, 11531 (1991); G. Ramirez-Santiago and J.V. José, Phys. Rev. Lett. 68, 1224 (1992); M. Benakli and E. Granato, Phys. Rev. B 55, 8361 (1997); G.S. Jeon, S.Y. Park, and M.Y. Choi, ibid. 55, 14088 (1997). E.H. Boubcheur and H.T. Diep, ibid. 58, 5163 (1998); S.E. Korshunov,
Phys. Rev. Lett. 88, 167007 (2002); E. Granato and D. Domínguez, Europhys. Lett. 63, 750 (2003).

11 E. Granato and M.P. Nightingale, Phys. Rev. B 48, 7438 (1993); S. Lee and K.-C. Lee, ibid. 49, 15184 (1994); J.V. José and G. Ramírez-Santiago, Phys. Rev. Lett. 77, 4849 (1996).

12 P. Olsson, Phys. Rev. Lett. 75, 2758 (1995).

13 K.H. Lee, D. Stroud, and J.S. Chung, Phys. Rev. Lett., 64 962 (1990); J.U. Free et al., Phys. Rev. B 41, 7267 (1990); K.H. Lee and D. Stroud, ibid. 43, 5280 (1991); B.J. Kim and P. Minnhagen, ibid. 60, 588 (1999).

14 M. Kvale and S.E. Hebboul, Phys. Rev. B 43, 3720 (1991); M.Y. Choi, ibid. 46, 564 (1992). See also M.Y. Choi and D.J. Thouless, ibid. 64, 014305 (2001).

15 B.J. Kim, P. Minnhagen, and P. Olsson, Phys. Rev. B 59, 11506 (1999).

16 S. Kim, B.J. Kim, and M.Y. Choi, Phys. Rev. B 52, 13536 (1995).

17 K. Binder, Phys. Rev. Lett. 47, 693 (1981).

18 See, e.g., E. Ott, Chaos (Cambridge, New York, 1993). Note that the FFJJA possesses many degrees of freedom, in contrast with the system studied mostly with regard to the Arnold tongue. 